\title{
Esophageal Dilatation in Children with Esophageal Strictures, Outcome and Safety at Single Center Experience
}

\author{
Abdulhamid Alhadab, Shaden N AlMahamed, Zahid Arein and \\ Abdelhai Hammo* \\ Department of Pediatric, Section of Pediatric Gastroenterology, Hepatology, and \\ Nutrition, King Fahad Specialist Hospital, Dammam, Saudi Arabia \\ *Corresponding Author: Abdelhai H Hammo, Department of Pediatric, Section of \\ Pediatric Gastroenterology, Hepatology, and Nutrition, King Fahad Specialist Hospital, \\ Dammam, Saudi Arabia.
} Received: April 09, 2020 Published: April 23, 2020 (C) All rights are reserved by Abdelhai Hammo., et al.

\begin{abstract}
Objective: The aim of this study is to assess the clinical response, nutritional outcome, and safety of endoscopic esophageal dilatation in pediatric esophageal stricture at KFSH-Dammam, Saudi Arabia.

Method: Charts of children between the age of 1 month and 16 years old with esophageal stricture who underwent endoscopic esophageal dilatation at King Fahad specialist Hospital-Dammam over a 5 years period from January 2014 until January 2019, was retrospectively reviewed. Patient's characteristics, procedure modality for stricture dilatation, clinical response, post dilatation catch up growth, and complications were reviewed and evaluated.

Result: A total of 17 patients (median age, 2.7 years were identified. The median follow-up period was 2 years. Post esophageal atresia repair stricture $(47.1 \%)$ and corrosive esophageal injury $(17.6 \%)$ were the leading cause of strictures. The main presenting symptoms were dysphagia (88.2\%) followed by vomiting (64.7\%) and then chronic cough (35.3\%). Majority of strictures were short segment $<2 \mathrm{~cm}$ in length $(70.6 \%)$. A total of 74 dilatation sessions were performed with an average of 4.3 sessions per patient. $70.8 \%$ underwent balloon dilatation. Complete response was achieved in (70.6\%) while partial response in (29.4\%). Complications of dilatation including esophageal perforation were reported in $1.3 \%$, and post-dilatation chest pain in $2.7 \%$. The mean weight-forage z-score improved significantly after ED therapy from $-1.8 \pm 0.79 \mathrm{SD}$ at presentation to $-1.01 \pm 0.85 \mathrm{SD}$ (P value $=0.003$ ).

Conclusion: Our single centre experience demonstrates that endoscopic dilatation is an effective and safe procedure for symptomatic esophageal stricture in children with different etiology. The choice of dilator device is based on multiple factors with minimal reported complications. Maintaining an age appropriate esophageal lumen by dilatation is very crucial to allow for satisfactory oral intake and promote catch-up growth which is the ultimate goal of dilatation.

Keywords: Esophageal Dilatation; Children; Esophageal Strictures (ES)
\end{abstract}

\section{Introduction}

Esophageal strictures (ES) in children are not uncommon condition especially in referring tertiary centers. In contrast to adults, the majority of causes are benign, including peptic esophageal stricture, post -surgical repair of esophageal atresia (EA), corrosive esophageal injury (CEI), congenital esophageal stenosis (CES) and eosinophilic esophagitis (EoE) [1].

The clinical presentation and the severity of symptoms are variable; depend on the location, type, and severity of the stricture. The main presenting symptoms are dysphagia, vomiting, and food regurgitation [2]. In addition, failure to thrive had been reported in as high as $50 \%$ of pediatric patient with esophageal stricture at diagnosis [3].

ES needs either endoscopic dilatation or surgical intervention to achieve clinical success and sufficient oral intake. Dilatation can be performed by using endoscopic balloon dilators (EBDs), which are currently being recognized as safe and effective method of ED in children [4]. Savary-Gillard Dilator (SGD) is another modality which has the advantage of lower cost and more availability in many centers. The end goal of the dilatation regardless of the procedure technique is always to achieve clinical response with age appropriate oral calories intake, allowing for catch up growth in pediatric patients.

\section{Aim of the Study}

This aim of our study is to evaluate the clinical response, nutritional outcome and the safety of endoscopic dilatation for symptomatic esophageal strictures in children at King Fahad Specialist Hospital-Dammam (KFSH-D) as a single center experience.

\section{Method and Patients}

This is a retrospective study of children between 1 month and 16 years of age, who referred to KFSH-D with symptomatic esophageal stricture over a period of 5 years (January 2014 to January 2019). 
Charts of 17 patients were reviewed retrospectively, including demographic data, clinical presentation, etiology of ES, stricture location by barium esophagogram (upper, middle or lower esophagus), stricture length ( $>2 \mathrm{~cm}$ long segment vs $<2 \mathrm{~cm}$ short segment), procedure modality used for ED, endoscopic finding, number of required sessions and follow up, use of adjuvant medications, catch up growth and post dilatation complications.

Dilatation procedure type and technique

Since there is no consensus regarding the use of either EBD or SGD in management of ES in children, in our institution the choice was mainly based on the type of stricture (simple, complicated, multiple level, tortuous strictures), length of stricture segment and the experience of pediatric gastroenterologist. All procedures were done under general anesthesia; written informed consent was obtained from the parents for the procedure after explaining benefits, risks and possible complications.

EBD procedure consists of using multi-diameter hydrostatic built-in wire guided balloon esophageal dilators (Boston Scientific, Ireland), the CRE balloon (controlled radial expansion) is $5.5 \mathrm{~cm}$ long, with a diameter ranging from $6 \mathrm{~mm}$ to $20 \mathrm{~mm}$. Endoscopy was initially performed to assess stricture location and luminal diameter, the balloon dilator then introduced through the working channel of the endoscope. The positioning of the balloon dilator at the waist of the stricture area was confirmed by fluoroscopy technique in few cases and estimated by direct vision via endoscopy in the rest of the cases. Each dilatation lasting for 30-60 seconds starting with balloon size of $1 \mathrm{~mm}$ above the lumen size and progressing to 2 more sizes above the resistance induced size, respecting the rule of three when it is possible. Favorable mucosal tears induced to allow for luminal stricture dilatation. SGD dilatation procedure were done under-fluoroscopy, and the dilator introduced via previously inserted guide-wire "endoscopically" or via gastrostomy tube (GT) for those who have previously inserted GT, same strategy of dilatation by BD were followed in SGD.

\section{Outcomes}

Success of dilatation was defined as a complete clinical response, absence of dysphagia and achievement of age-appropriate feeding without the need for further dilatation. However, the partial improvement of the symptoms and the oral intake after completing 5 sessions of dilatation is referred to partial clinical response. Failure of clinical response or refractory stricture is stated for failure to obtain age-appropriate oral feeding after a maximum of five dilation sessions with maximal four-week intervals.

\section{Assessment of nutritional status}

The nutritional status of the patients was assessed at presentation prior to ED, and after clinical response. The nutritional parameter assessed by weight-for-age z-score. The weight-for-age z-score was calculated using WHO Anthro. v.3.2.2 software in patients who were younger than 5 years and the WHO Anthropometric Plus v.1.0.4 software in patients who were aged 5 - 10 years. One patient above 10 year we used who 2007 macro package for
$\mathrm{R}$, hosted on a Shiny server which used to calculate anthropometric Z-scores for children aged 5 - 19 years based on the 2007 World Health Organization 'reference' charts and LMS tables. Failure to thrive (FTT) was defined as weight-for-age-z-score of $<-2$ SD below the mean.

\section{Statistical analysis}

- Methodology of study design, participants recruitment and data collection.

- $\quad$ Data were analyzed using an IBM personal computer with Statistical Package of Social Science (SPSS) version 20. Normality of continuous variables was examined and tested using Shapiro-Wilk test. Continuous variables were presented in the form of median and inter quartile range (IQR), binary and categorical variables were presented in the form of numbers and percentages (\%).

- $\quad$ This study was approved by Institutional Research Ethics board at KFSH-D (IRB\# PED0305).

\section{Result}

The patient's characteristics and demographics data are summarized in table 1 . A total of 17 patients were identified during study period. There were 12 males (70.6\%) and 5 females $(29.4 \%)$. The median age at presentation was 2.7 years (range, 0.5 - 16 years), and youngest age at dilatation was seven months. Fifteen patients (88.2\%) presented with dysphagia, being the most common presenting symptom in our patients and followed by vomiting (64.7\%). Six patients presented with chronic cough (35.3\%), drooling of saliva in two patients (11.8\%) and food impaction in one patient. Around one third of the patients (35.3\%) have failure to thrive (FTT) at presentation. The median follow-up period for each patient was 2 years (range, 10 months to 5 years).

\begin{tabular}{|l|c|}
\hline Characteristic & Outcome No (\%) \\
\hline Gender & $12(70.6)$ \\
Male & $5(29.4)$ \\
\hline Age at presentation (years) & \\
Median & 2.7 \\
Range & $0.5-16$ \\
\hline Etiology of ES & \\
Post-EA repair & $8(47.1)$ \\
Corrosive esophageal injury & $3(17.6)$ \\
Eosinophilic esophagitis & $2(11.8)$ \\
Peptic stricture & $2(11.8)$ \\
Congenital esophageal stenosis & $1(5.9)$ \\
Post radiation therapy & $1(5.9)$ \\
\hline Clinical presentation & \\
Dysphagia & $15(88.2)$ \\
Vomiting & $11(64.7)$ \\
Chronic Cough & $6(35.3)$ \\
Drooling & $2(11.8)$ \\
Food impaction & $1(5.9)$ \\
\hline
\end{tabular}




\begin{tabular}{|l|c|}
\hline FTT & \\
Z score $<-2$ SD of mean & $6(35.3)$ \\
\hline Site of stenosis & \\
Upper & $6(35.3)$ \\
Middle & $7(41.2)$ \\
Lower & $3(17.6)$ \\
Multiple & $1(5.9)$ \\
\hline Length of stenosis & \\
Short segment $<2 \mathrm{~cm}$ & $12(70.6)$ \\
Long segment $>2 \mathrm{~cm}$ & $5(29.4)$ \\
\hline
\end{tabular}

Table 1: Demographic data and patients characteristics.

In regard of the etiology of the stricture, Post EA repair stricture (47.1\%) and CEI (17.6\%) were the leading cause of ES for our patients. Other causes are summarized in table 1.

Among the 17 patients, stricture at the upper esophagus was found in 6 patients $(35.3 \%)$, in the middle third esophagus in 7 $(41.2 \%)$ and in the lower third esophagus in $3(17.6 \%)$ patients. One patient had stricture at multiple levels which was secondary to corrosive esophageal injury. Twelve (70.6\%) patients had short segment esophageal stricture $<2 \mathrm{~cm}$, while 5 (29.4\%) had long segment stricture $>2 \mathrm{~cm}$.

The type of procedure modality, clinical response, and complications are summarized in table 2 , A total of 74 sessions of dilatation were performed in 17 patients, the median number of dilatation sessions per patient was 4 (Range, 2 - 13). 12 patients (70.6\%) underwent EBD, while SGD dilatation was used in 5 patients (29.4\%). Triamcinolone was used only twice during the study period in poor responder stricture and was helpful in facilitating the later on response [5]. Twelve patients (70.6\%) achieved complete clinical response with improvement of swallowing and nutritional status while five patients (29.4\%) experienced partial clinical response and no patient had dilatation failure according to the outcome criteria.

\begin{tabular}{|c|c|}
\hline & No. (\%) \\
\hline $\begin{array}{l}\text { Type of dilatation procedure } \\
\text { - } \quad \text { EBD } \\
\text { - } \quad \text { Savary-Gilard dilatation }\end{array}$ & $\begin{array}{c}12(70.6) \\
5(29.4)\end{array}$ \\
\hline $\begin{array}{l}\text { Number of sessions } \\
\text { - } \quad \text { Total No. } \\
\text { - } \quad \text { Median/patient (Range) } \\
\text { - } \quad \text { EBD (mean) } \\
\text { - } \quad \text { SGD (mean) }\end{array}$ & $\begin{array}{c}74 \\
4(2-13) \\
45(3.7) \\
29(5.8)\end{array}$ \\
\hline $\begin{array}{l}\text { Use of Adjuvant medication (per session) } \\
\text { - } \quad \text { Steroid (Triamcinolone) } \\
\text { - } \quad \text { Mitomycin } \\
\end{array}$ & $\begin{array}{c}2(2.7) \\
0\end{array}$ \\
\hline 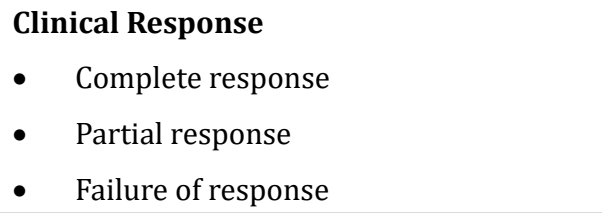 & $\begin{array}{c}12(70.6) \\
5(29.5) \\
(0)\end{array}$ \\
\hline
\end{tabular}

\begin{tabular}{|c|c|}
\hline Catch up growth after ED & $5(83.3)$ \\
\hline $\begin{array}{l}\text { Complication* } \\
\text { - } \quad \text { Esophageal Perforation } \\
\text { - } \\
\text { - } \\
\text { Post-dilatation bleeding } \\
\text { Post-dilatation pain } \\
\end{array}$ & $\begin{array}{c}1(1.3) \\
0(0) \\
2 \dagger(2.7)\end{array}$ \\
\hline $\begin{array}{l}\text { Duration of follow up } \\
\text { - } \quad \text { Median (year) (SD) }\end{array}$ & $2 \pm 1.2$ \\
\hline $\begin{array}{l}\text { *: No of complications to total No of dilata- } \\
\text { tions } \\
\text { †: Post procedure chest pain that required } \\
\text { pain killer and/or further observation or } \\
\text { evaluations. }\end{array}$ & \\
\hline
\end{tabular}

Table 2: Procedures, clinical response and complications.

Out of 74 sessions of ED, transmural esophageal perforation and Post dilatation chest pain occurred once $(1.3 \%)$ and twice $(2.7 \%)$, respectively. Patient with post-dilatation chest pain managed with analgesic and observation only. Perforation occurred during BED in one patient with CEI and tortuous long segment stricture and was managed surgically with thoracotomy and esophageal repair. No post dilatation bleeding was seen in our patient.

Additional surgical interventions were needed in 3 patients ( $\mathrm{n}=$ 3, 17.6\%), 2 patients with CEI required gastrostomy tube insertion for feeding and one patient with peptic stricture underwent Nissen fundoplication for associated hiatal hernia.

The mean weight-for-age z-score of the 17 patients at diagnosis was $-1.7 \pm 0.83 \mathrm{SD}$. Failure to thrive with A z-score of $<-2 \mathrm{SD}$ was found in six patients (35.3\%). Two out of the 17 patients underwent gastrostomy feeding tube. Weight records for nutritional assessment were calculated for 15 patients excluding patients who underwent gastrostomy. The mean weight-for-age z-score of the 15 patients at diagnosis was $-1.8 \pm 0.79$ SD which increased significantly after ED therapy on their last visit to $-1.01 \pm 0.85$ SD (P val$\mathrm{ue}=0.003)$. The proportion of $\mathrm{z}$ scores less than $<-2 \mathrm{SD}$ decreased from $40 \%(6 / 15)$ to $6 \%(1 / 15)$ after ED (Figure 1$)$.

\begin{tabular}{|l|c|c|c|}
\hline & $\begin{array}{c}\text { Weight-for-age-Z- } \\
\text { score before ED } \\
\text { mean } \pm \text { (SD) }\end{array}$ & $\begin{array}{c}\text { Weight-for-age- } \\
\text { Z-score after ED } \\
\text { Mean } \pm \text { (SD) }\end{array}$ & P value \\
\hline $\begin{array}{c}\text { Overall } \\
(\mathrm{n}=15)\end{array}$ & $-1.8-+(0.79)$ & $-1.01-+(0.85)$ & 0.003 \\
\hline
\end{tabular}

Table 3: Nutritional assessment.

\section{Discussion}

Esophageal strictures in children have multiple etiologies. The incidence of the different etiologies varies between countries. In developing countries, caustic injuries are more frequent [6]. In our study Post-EA repair stricture is the most common cause of referral for esophageal dilatation in our center, followed by CEI, other causes like eosinophilic esophagitis, peptic stricture, and congenital stricture were account for less percentage. Previous studies showed the Post EA repair strictures and CEI as the two most com- 


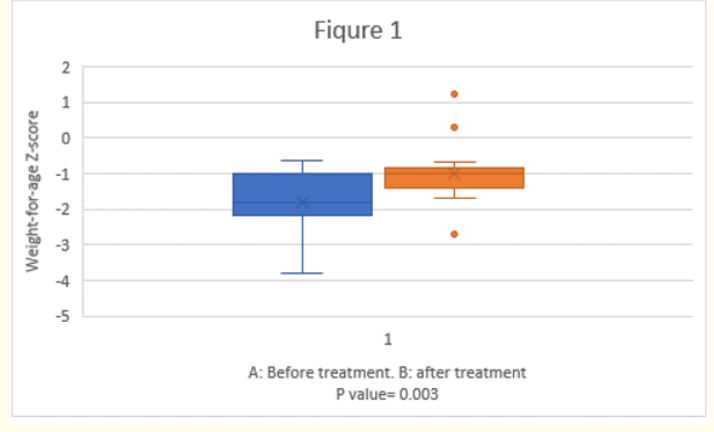

Figure 1

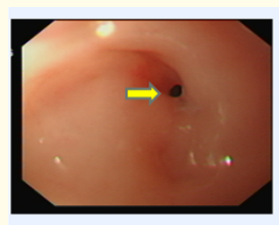

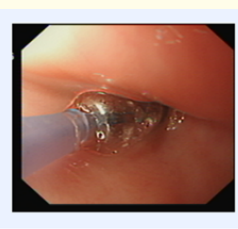

B

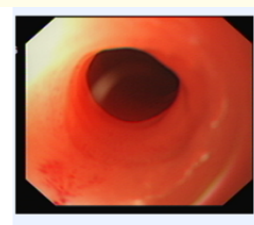

Figure 2: Patient with EoE and ES. A) Pinhole esophageal stricture (arrow). B) The balloon dilatation. C) Post dilatation mucosal tears.

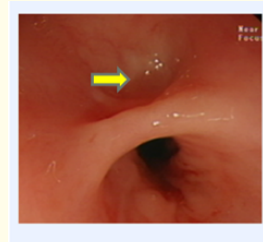

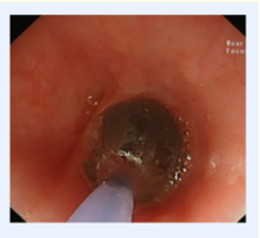

B

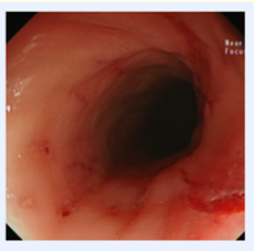

C
Figure 3: Patient with ES secondary to post EA repair. Narrowed lumen at stricture level with secondary sided pouch (arrow). B) The balloon dilatation. C) Post dilatation with mucosal tears and dilated lumen.

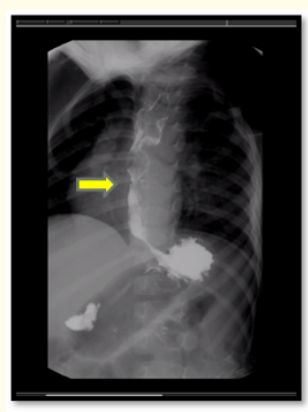

A

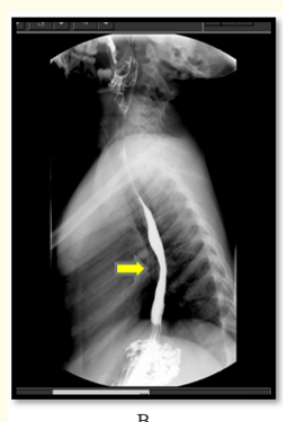

Figure 4: Barium Esophagogram for patient with ES secondary to EoE. (A) Short segment Stricture at mid esophagus (arrow).

(B) Post-dilatation stricture improvement (arrow).

mon indication for esophageal dilatation in children [1,4,7-10]. Study from Saudi Arabia included 43 children, reported the postEA repair strictures to be $(32.6 \%)$ as the most common etiology, while CEI account for only $5 \%$ of the cases [11].
Endoscopic dilatation is considered the treatment of choice in children with symptomatic esophageal strictures before any surgical intervention [12]. SGD and EBD with or without fluoroscopic guidance are the two strategies used for esophageal dilatation. SGD exerts an abrupt shearing axial force during the repeated insertion of the SGD into stricture that may increase the risk of esophageal trauma, while balloon dilators (CRE) which pass over a guide wire or through the channel of the endoscope have the advantage of applying a gradual uniform radial force on the stricture area in which both the diameter of the balloon and the dilatation pressure can be precisely controlled as well as accurate placement of balloon across stricture with visualization of the dilatation process $[4,13]$.

Increasing of esophageal diameter which observed during endoscopy is a direct objective measure to assess dilation success while resolution of stricture waist by esophagogram is another indirect measure. Furthermore, the improvement of oral intake and weight gain are important parameters for assessing the clinical response and dilation success $[11,13,14]$.

The choice of procedure modality for dilatation, either EBD or SGD is mainly depends on the stricture type, length of stricture, device availability, and the operator experience/preference. In recent studies, EBD is preferred than SGD as a safe and effective method of treating a variety of esophageal strictures in children with overall success rates range from $60 \%$ to $100 \%$, depending on the cause and criteria used to define the effectiveness, with lowest success rate in corrosive and long stricture $[8,15,16]$. Lan-Lan Geng., et al. reported (60\%) success rate of EBD for patient with corrosive esophageal injury with significant result in short segment as compared to long segment stricture, Similar result also reported by Gundogdu., et al. with (70\%) overall success rate and (91\%) in strictures less than 5 cm in length $[6,16]$.

In our patients, EBD was the main procedure used for dilatation $(70.6 \%)$ while SGD was used only in $(29.4 \%)$ of cases. The overall success rate was $(70 \%)$ for those who achieve complete response without the need for further dilatation, while $30 \%$ of the patients had partial response, and there was no patient with treatment failure. in general, our success rate was similar and comparable to previously reported studies in children. The median number of dilatation sessions per patient which needed to achieve a complete response was 4 sessions per patient, patients who managed by SGD were found to require more sessions (average of 5.7 per patient) as compared to 3.7 session for patients with EBD, this finding could be related to the underlying stricture etiology as three out of the five patients with SGD were secondary to corrosive esophageal injury which is usually more difficult to dilate. Furthermore, our finding indirectly might indicate the superior effectiveness of the balloon technique for short segment stricture over SGD in our sample. Previous studies reported a median number of 2 - 5 dilatation sessions per patient to achieve a complete/satisfactory response with more session for patient with corrosive esophageal injury $[1,7,11,17,18]$. Studies from Saudi Arabia in pediatric patients with esophageal stricture reported a median of 4 sessions of dilations per patient were required, and caustic injuries induced stricture required the highest number of dilatation sessions in one study, while those 
with EoE required 2 sessions on average of SGD to achieve a clinical response in another one [11,14]. Other studies on pediatric population reported an average of 20 sessions of SGD per patient in general with higher number 34 sessions per patient with corrosive esophageal injury $[1,18]$.

Esophageal stricture has been reported in children on chemotherapy for hematologic malignancy, secondary to deep mucositis and full-thickness inflammation and fibrosis [19,20]. ED and stenting represent the first option in such cases however esophageal substitution could be an option in case of recurrence [4]. In our experience we had only one case post chemo and radiotherapy, and ES was responded completely to EBD without the need for stenting or substitution.

Catch up growth is considered as important predictor for clinical response post dilatation. Our study indicates that those who had FTT secondary to esophageal stricture and feeding difficulties showed significant improvement in their nutritional status (weight-for-age z-score) in response to ED therapy. The present study is one of few studies which evaluate the therapeutic effect of ED on nutritional outcome in addition to the clinical effectiveness of the dilatation procedure. Chang., et al. reported a 5-year retrospective study on 50 pediatric patients with significant increased weight-for-age $\mathrm{z}$ scores in all the patients receiving EBD, particularly in those with short strictures in the middle esophagus, and Thorsten Vowinkel., et al. reported that BED helps to promote catch-up growth and body weight in 12 patients with dystrophic epidermolysis bullosa all were unable to meet their nutritional needs orally due to secondary esophageal stricture $[7,21,22]$.

Esophageal perforation, bleeding and post-procedure chest pain are frequently encountered as complications of ED. Esophageal perforation is the most serious complication post dilatation, and its rate has been estimated as (5.6\%) for SGD and (0.28 - 4\%) for EBD in previous studies [8,16,23]. In our patients we had only 1 event of esophageal perforation (1.3\%), post balloon dilatation in a patient with long tortious esophageal stricture secondary to corrosive esophageal injury which required surgical repair and SGD later on. In the literature the risk of perforation was reported to be higher in case of corrosive injury induced stricture especially during EBD (16 - 33\%) and post dilatation perforation was also reported with higher rate $(10 \%-44 \%)$ in case of congenital stricture like tracheobronchial remnant $[4,16,17,24]$. Postprocedural chest pain occurred once in two patients $(2.7 \%)$ both after SGD, and managed by analgesic. A recent Systematic Review included 461 patients showed that the postprocedural chest pain was lower in patient received balloon dilatation as compared to SGD [4].

\section{Conclusion}

Our single centre experience demonstrates that endoscopic dilatation is an effective and safe procedure for symptomatic esophageal stricture in children with different etiology. The choice of dilator device is based on multiple factors with minimal reported complications. Maintaining an age appropriate esophageal lumen by dilatation is very crucial to allow for satisfactory oral intake and promote catch-up growth which are the ultimate goal of dilatation.

\section{Acknowledgment}

We would like to thank Dr Mushari Al Amer for contribution and efforts in statistical data analysis.

\section{Bibliography}

1. Saleem MM. "Acquired oesophageal strictures in children: Emphasis on the use of string-guided dilatations". Singapore Medical Journal 50.1 (2009): 82-86.

2. Josino IR., et al. "Endoscopic Dilation with Bougies versus Balloon Dilation in Esophageal Benign Strictures: Systematic Review and Meta-Analysis". Gastroenterology Research and Practice (2018): 1-9.

3. Lakhdar-Idrissi M., et al. "Esophageal endoscopic dilations". Journal of Pediatric Gastroenterology and Nutrition 54.6 (2012): 744-747.

4. Dall'Oglio L. "Endoscopic management of esophageal stenosis in children: New and traditional treatments". World Journal of Gastrointestinal Endoscopy 8.4 (2016): 212.

5. Mark Anthony A De Lusong., et al. "Management of esophageal caustic injury". World Journal of Gastrointestinal Pharmacology and Therapeutics 8.2 (2017): 90-98.

6. Khanna S and Khanna S. "Management of benign oesophageal strictures in children". Indian Journal of Otolaryngology and Head and Neck Surgery 60.3 (2008): 218-222.

7. Chang $\mathrm{CH}$., et al. "Clinical and nutritional outcome of pediatric esophageal stenosis with endoscopic balloon dilatation". Pediatrics and Neonatology 60.2 (2019): 141-148.

8. Pieczarkowski S., et al. "Endoscopic therapy of oesophageal strictures in children - A multicentre study". Przegląd Gastroenterologiczny 11.3 (2016): 194-199.

9. Chamorro CC., et al. "Dilatación endoscópica con balón de estenosis esofágica en niños". Cirugía Pediátrica 26.3 (2013): 106-111.

10. Antoniou D., et al. "Anastomotic strictures following esophageal atresia repair: A 20-year experience with endoscopic balloon dilatation". Journal of Pediatric Gastroenterology and Nutrition 51.4 (2010): 464-467.

11. Al Sarkhy AA., et al. "Efficacy and safety of endoscopic dilatation in the management of esophageal strictures in children". Saudi Medical Journal 39.8 (2018): 787-791.

12. Ghiselli A., et al. "Endoscopic dilation in pediatric esophageal strictures: A literature review". Acta BioMedica 89.1 (2018): 27-32.

13. Alshammari J., et al. "Endoscopic balloon dilatation of esophageal strictures in children". International Journal of Pediatric Otorhinolaryngology 75.11 (2011): 1376-1379. 
14. Al-Hussaini A. "Savary dilation is safe and effective treatment for esophageal narrowing related to pediatric eosinophilic esophagitis". Journal of Pediatric Gastroenterology and Nutrition 63.5 (2016): 474-480.

15. Fang S Bin. "Endoscopic balloon dilatation in pediatric patients with esophageal strictures: From the past to the future". Pediatrics and Neonatology 60.2 (2019):119-120.

16. Jayakrishnan VK and Wilkinson AG. "Treatment of oesophageal strictures in children: A comparison of fluoroscopically guided balloon dilatation with surgical bouginage". Pediatric Radiology 31.2 (2001): 98-101.

17. Youn BJ., et al. "Balloon dilatation for corrosive esophageal strictures in children: Radiologic and clinical outcomes". Korean Journal of Radiology 11.2 (2010): 203-210.

18. Karaman I., et al. "Evaluation of 968 children with corrosive substance ingestion". Indian Journal of Critical Care Medicine 19.12 (2015): 714-718.

19. Kelly K., et al. "Esophageal strictures during treatment for acute lymphoblastic leukemia". Journal of Pediatric Hematology/Oncology 32.2 (2010): 124-127.

20. Temiz A., et al. "Long-term management of corrosive esophageal stricture with balloon dilation in children". Surgical Endoscopy 24.9 (2010): 2287-2292.

21. Chang CF., et al. "Endoscopic balloon dilatation for esophageal strictures in children younger than 6 years: Experience in a medical center". Pediatrics and Neonatology 52.4 (2011): 196202.

22. Vowinkel T., et al. "A two-step multidisciplinary approach to treat recurrent esophageal strictures in children with epidermolysis bullosa dystrophica". Endoscopy 58 (2015): 541-544.

23. Lan LCL., et al. "Endoscopic Balloon Dilatation of Esophageal Strictures in Infants and Children: 17 Years' Experience and a Literature Review". Journal of Pediatric Surgery 38.12 (2003): 1712-1715.

24. Terui K. "Endoscopic management for congenital esophageal stenosis: A systematic review". World Journal of Gastrointestinal Endoscopy 7.3 (2015): 183.

\section{Assets from publication with us}

- Prompt Acknowledgement after receiving the article

- Thorough Double blinded peer review

- Rapid Publication

- Issue of Publication Certificate

- High visibility of your Published work

Website: www.actascientific.com

Submit Article: www.actascientific.com/submission.php

Email us: editor@actascientific.com

Contact us: +919182824667 